\title{
MicroRNA-320a inhibition decreases insulin-induced KGN cell proliferation and apoptosis by targeting PCGF1
}

\author{
LEI YANG ${ }^{1}$, YAN LI ${ }^{1}$, XIAOJING WANG ${ }^{2}$, YULING LIU ${ }^{2}$ and LINGZHU YANG ${ }^{1}$ \\ ${ }^{1}$ Department of Obstetrics and Gynecology Surgery, The First Affiliated Hospital of Zhengzhou \\ University, Zhengzhou, Henan 450052; ${ }^{2}$ Department of Obstetrics and Gynecology Surgery, The \\ Second Affiliated Hospital of Zhengzhou University, Zhengzhou, Henan 450014, P.R. China
}

Received June 3, 2016; Accepted May 22, 2017

DOI: $10.3892 / \mathrm{mmr} .2017 .7270$

\begin{abstract}
MicroRNAs (miRNAs) are widely involved in regulation of cellular processes of polycystic ovary syndrome (PCOS). However, the function of miR-320a in PCOS remains unclear. The present study aimed to explore the effect of miR-320a on PCOS cell proliferation and apoptosis following treatment with insulin, and to clarify the underlying mechanism. PCOS tissues and corresponding normal tissues were collected from 16 female patients with PCOS. KGN cells were pre-treated with insulin, and KGN cells were transfected with ASO-miR-320a, miR-320a mimics and polycomb group ring finger 1 (PCGF1) overexpression plasmids. Expressions of miR-320a and PCGF1 were detected using the reverse transcription-quantitative polymerase chain reaction (RT-qPCR). Dual-Luciferase reporter assays were performed to investigate the target gene of miR-320a. MTS, colony formation and flow cytometry assays were performed to determine cell viability, colony formation, and apoptosis, respectively. Furthermore, mRNA and protein expression levels of B-cell lymphoma 2 apoptosis regulator (Bcl-2) and Bcl-2 associated protein $\mathrm{X}$ apoptosis regulator (Bax) were examined using RT-qPCR and western blotting. The results demonstrated that miR-320a expression was significantly increased in PCOS tissues compared with normal tissues. Moreover, miR-320a was upregulated in insulin-induced cells in a dose-dependent manner. Inhibition of miR-320a suppressed insulin-induced cell viability and colony formation, and promoted apoptosis. Luciferase reporter assays demonstrated that PCGF1 was
\end{abstract}

Correspondence to: Dr Yuling Liu, Department of Obstetrics and Gynecology Surgery, The Second Affiliated Hospital of Zhengzhou University, 2 Jingba Road, Zhengzhou, Henan 450014, P.R. China E-mail: liuyuling9111@126.com

Dr Lingzhu Yang, Department of Obstetrics and Gynecology Surgery, The First Affiliated Hospital of Zhengzhou University, 1 Jianshe East Road, Zhengzhou, Henan 450052, P.R. China

E-mail: yanglingzhu811@sina.com

Key words: polycystic ovary syndrome, polycomb group ring finger 1, insulin, microRNA-320a, cell growth a target of miR-320a. Additionally, PCGF1 overexpression inhibited cell viability and colony formation and promoted apoptosis. Additionally, the mRNA and protein levels of Bcl-2 were inhibited by miR-320a suppression and PCGF1 overexpression, while Bax expression was promoted by them in insulin-induced cells. The results of the present study demonstrated that miR-320a inhibition decreased insulin-induced KGN cell proliferation and apoptosis by targeting PCGF1. These data indicated that miR-320a may serve as a potential diagnostic biomarker for PCOS.

\section{Introduction}

Polycystic ovary syndrome (PCOS) is a common clinical endocrine disorder in women with high prevalence and socioeconomic costs, affecting $\sim 10 \%$ women of reproductive age (1-3). Anovulatory infertility and compensatory hyperinsulinemia are common symptoms of PCOS $(3,4)$. Women with PCOS have been demonstrated to be at higher risk for type 2 diabetes, with $>60 \%$ of women with PCOS diagnosed with insulin resistance (4).

The pathogenesis of PCOS is complex and the mechanisms remain unclear (5). The number of growing follicles in PCOS ovaries is doubled, and there is an active relationship between granulosa cell proliferation and growing follicles (4). Numerous studies have demonstrated that PCOS is associated with genetic factors including microRNAs (miRNAs or miRs) (4,6). miRNAs are involved in the development and progression of many human diseases, including some which have been demonstrated to be crucial for the pathogenesis of PCOS, including miR-93 (4), miR-21 (7) and miR-320 (8). miR-93 is a biomarker identified to be differentially expressed in PCOS adipose tissues (4). miR-93 promotes ovarian granulosa cell proliferation by targeting solute carrier family 2 member 4 (SLC2A4, also known as GLUT4) or/and cyclin dependent kinase inhibitor 1A (CDKN1A), and insulin administration has been demonstrated to upregulate miR-93 expression and cell proliferation $(3,4)$. A negative relationship has been revealed between expression of miR-93 and CDKN1A, and miR-93 overexpression and knockdown of CDKN1A are demonstrated to promote cell growth and cell cycle progression in KGN granulosa cells (3). miR-320a is a cancer related miRNA that is important in disease progression 
and pathogenesis and modulates several target genes, including integrin subunit beta 3 (ITGB3) (9), ras-related C3 botulinum toxin substrate 1 (Rac1) (10), and mitogen-activated protein kinase 1 (MAPK1) (11), which participates in various signaling pathways in diseases (12). However, the effect of miR-320a on cell function of KGN granulosa cells has previously been remained unclear.

The present study aimed to investigate the effect of miR-320a on PCOS cell function, using PCOS samples collected from 16 patients and KGN granulosa cells. miR-320a expression in PCOS tissues and insulin treated KGN cells was detected using reverse transcription-quantitative polymerase chain reaction (RT-qPCR). Cell functions, including cell viability, cell colony formation and cell apoptosis, were detected in insulin treated or insulin plus miR-320a antisense oligonucleotide/plasmid of miR-320a predicted target. The present study therefore expands what is known about the effect of miR-320a on PCOS granulosa cells and presents it as a potential biomarker for PCOS.

\section{Materials and methods}

Collection of clinical samples. Between January 2013 and December 2014, a total of 16 female patients with PCOS were recruited from The Second Affiliated Hospital of Zhengzhou University (Zhengzhou, China). The selection of PCOS samples was primarily based on the clinical criteria for PCOS, the revised Rotterdam European Society of Human Reproduction and Embryology/American Society for Reproductive Medicine criteria (2003). The baseline information of patients, including fasting insulin levels, was collected (3). PCOS samples and corresponding non-tumor normal tissues were obtained from patients who had undergone laparoscopic surgery, and were assigned to two groups according to the insulin level: High or normal. The present study was approved by the Research Ethics Committee of The Second Affiliated Hospital of Zhengzhou University. Informed and written consent was obtained from all patients prior to surgery. Samples were cut into pieces and stored at $-80^{\circ} \mathrm{C}$ until required.

Cells, cell culture, insulin induction and transfection. Human granulosa-like tumor cell line KGN was obtained from Sciencell Research Laboratories, Inc. (Carlsbad, CA, USA) and grown in DMEM/F12 medium (Invitrogen; Thermo Fisher Scientific, Inc., Waltham, MA, USA) with $10 \%(\mathrm{v} / \mathrm{v})$ fetal bovine serum (FBS; Sciencell Research Laboratories, Inc.), $100 \mathrm{U} / \mathrm{ml}$ penicillin $\mathrm{G}$ and $0.1 \mathrm{mg} / \mathrm{ml}$ streptomycin sulfate (Invitrogen; Thermo Fisher Scientific, Inc.) at $37^{\circ} \mathrm{C}$ with $5 \% \mathrm{CO}_{2}$. Cells were seeded into 6 -well plates at a density of $2 \times 10^{5}$ cells per well and treated with recombinant human (rh) insulin (Roche Diagnostics, Basel, Switzerland) at 0, 1, 10 or $100 \mathrm{ng} / \mathrm{ml}$ for $24 \mathrm{~h}$. ASO-miR-320a was synthesized by Shanghai GenePharma Co., Ltd. (Shanghai, China), and Polycomb group ring finger 1 (PCGF1) was obtained from the laboratory of the First Affiliated Hospital of Zhengzhou University (Zhengzhou, China) and was cloned into the pcDNA3.1 vector (Sangon Biotech Co., Ltd., Shanghai, China) for overexpression of PCGF1. KGN cell transfections were performed using Lipofectamine 2000 reagent (Invitrogen;
Thermo Fisher Scientific, Inc.), according to the manufacturer's protocol, for $72 \mathrm{~h}$.

MTS assay. Cell viability was examined using MTS assays (3). Briefly, cells transfected with ASO-miR-320a or PCGF1 expression plasmids were plated on 96-well plates at a density of 1,500 cells/well for 24,48 and $72 \mathrm{~h}$. For insulin treatment, rh insulin at $0,1,10,100 \mathrm{ng} / \mathrm{ml}$ cells were placed into 96-well plates, then $20 \mu \mathrm{l}$ MTS (Sigma-Aldrich; Merck KGaA, Darmstadt, Germany) was added to each well and incubated for a further $3 \mathrm{~h}$ at $37^{\circ} \mathrm{C}$ in a humidified $5 \% \mathrm{CO}_{2}$ and $95 \%$ air atmosphere. The absorbance at $490 \mathrm{~nm}$ for each sample was measured using a microplate reader (Bio-Rad Laboratories, Inc., Hercules, CA, USA).

Colony formation assays. KGN cells (600 cells per well) were plated in 12-well plates for 14 days, with the medium replaced every 3-5 days. Cells were stained with crystal violet and the number of colonies with more than 50 cells was counted. Each treatment was performed in triplicate.

Cell apoptosis assay. KGN cells at $5 \times 10^{5}$ cells/well were cultured and were stained with fluorescein isothiocyanate (FITC)-conjugated Annexin V and propidium iodide (PI) and incubated in a dark at room temperature for $30 \mathrm{~min}$ and subjected to the flow cytometry according to the manufacturer's protocol (Beijing Biosea Biotechnology Co., Ltd., Beijing, China). The percentage of apoptotic cells was detected using a FACS Sort Flow Cytometer (BD Biosciences, Franklin Lakes, NJ, USA) at $488 \mathrm{~nm}$.

Luciferase reporter assay. Targetscan (www.targetscan.org) and microRNA database (www.microrna.org) were used to predict that PCGF1 was a potential target of miR-320a. Dual-luciferase reporter assays were performed using the methods reported by Jiang et al (3). In brief, KGN cells were cultured and transfected with reporter vector PCGF1-3'UTR wild type (WT) and mutant (MUT), together with miR-320a mimics, ASO-miR-320a or negative control using Lipofectamine 2000 (Invitrogen) for 48 h. Cells were then harvested and the Dual-luciferase Reporter Assay System (Promega Corporation, Madison, WI, USA) was used to assay the luciferase activity, according to the manufacturer's instructions.

Western blot analysis. Following incubation under various conditions, $1 \times 10^{5} \mathrm{KGN}$ cells were harvested, washed and re-suspended in radio immunoprecipitation assay cell lysis solution (Beyotime Institute of Biotechnology, Haimen, China). Protein concentrations were determined using the bicinchoninic acid assay kit (Pierce; Thermo Fisher Scientific, Inc, ). Samples were separated by $10 \%$ SDS-PAGE and transferred onto polyvinylidene difluoride membranes (Merck KGaA). Membranes were blocked in 5\% bovine serum albumin (Roche Diagnostics) in TBS-Tween 20 at room temperature for $1 \mathrm{~h}$ and probed with antibodies against PCGF1 (cat. no. ab84108), B-cell lymphoma 2 apoptosis regulator (Bcl-2; cat. no. ab59348), Bcl-2 associated protein $\mathrm{X}$ apoptosis regulator (Bax; cat. no. ab53154) and GAPDH (cat. no. ab37168) (all Abcam, Cambridge, UK) at a dilution of 
1:1,000 overnight at $4^{\circ} \mathrm{C}$. Membranes were then incubated with horseradish peroxidase-conjugated secondary antibodies (cat. no. ab205718; Abcam) at a dilution of 1:5,000 for $1 \mathrm{~h}$ at room temperature, then immunoreactive protein bands were visualized using enhanced chemiluminescence (GE Healthcare, Chicago, IL, USA) and analyzed using Image Gauge 4.0 software (Fujifilm Corporation, Tokyo, Japan).

$R T-q P C R$. Total RNA was extracted and prepared using TRIzol reagent (Thermo Fisher Scientific, Inc.) according to the manufacturer's protocol. For detection of miR-320a (forward: 5'-GGGAAAAGCTGGGTTGAGA-3'; reverse: 5'-CAGTGC GTGTCGTGGAGT-3') and the U6 control (forward: 5'-CTT CGGCAGCACATATACT-3'; reverse: 5'-AAAATATGG AACGCTTCACG-3'), RNA was reverse transcribed using a PrimeScript RT reagent Kit (Takara Bio, Inc., Otsu, Japan). For detection of PCGF1 (forward: 5'-GTATGTCCGATGTTCTGT TAG-3'; reverse: 5'-GCCATATCTGCTTCATTGTC-3') and control GAPDH (forward: 5'-GGAGCGAGATCCCTCCAA AAT-3'; reverse: 5'-GGCTGTTGTCATACTTCTCATGG-3'), reverse transcription of total RNA was performed using a PrimeScript RT reagent kit with gDNA Eraser (Takara). qPCR was performed using a SYBR green PCR master mix (Takara Bio, Inc.) with a LightCycler Real-Time PCR System (Roche Applied Science, Penzburg, Germany). The reaction conditions were as follows: Initial denaturation was performed at $95^{\circ} \mathrm{C}$ for $10 \mathrm{~min}$, followed by 30 cycles of denaturation for $30 \mathrm{sec}$ at $95^{\circ} \mathrm{C}$, annealing at $60^{\circ} \mathrm{C}$ for $1 \mathrm{~min}$, extension at $72^{\circ} \mathrm{C}$ for $15 \mathrm{sec}$, and the final extension was at $72^{\circ} \mathrm{C}$ for $10 \mathrm{~min}$. These data were calculated by the $2^{-\Delta \Delta \mathrm{Ct}}$ method (13).

Statistical analysis. Values are expressed as the mean \pm standard deviation (SD) of three independent experiments. Statistical analyses were performed using GraphPad Prism 5 (GraphPad Software, Inc., La Jolla, CA, USA). The $\mathrm{P}$-values were calculated using a one-way analysis of variance followed by Duncan's multiple-range test. $\mathrm{P}<0.05$ was considered to indicate a statistically significant difference.

\section{Results}

miR-320a expression is increased in PCOS tissues. The expression levels of miR-320a in PCOS samples and corresponding normal tissues from 16 patients with PCOS were examined by RT-qPCR. The results demonstrated that miR-320a expression was significantly higher in PCOS samples than in the normal tissues $(\mathrm{P}<0.05$; Fig. 1A). Further study revealed that that miR-320a expression was significantly higher in the PCOS samples from patients with high insulin levels compared with the normal group $(\mathrm{P}<0.01$; Fig. 1B). It was therefore hypothesized that miR-320a expression was insulin-dependent. To test this hypothesis, miR-320a expression was detected in KGN cells treated with gradually increasing concentrations of rh insulin. As predicted, miR-320a expression increased in a concentration-dependent manner $(\mathrm{P}<0.05$; Fig. 1C).

miR-320a inhibition reduces insulin-induced KGN cell viability, colony formation and induces cell apoptosis. To investigate the effect of miR-320a on KGN cell function, KGN cells were treated with either $100 \mathrm{ng} / \mathrm{ml} \mathrm{rh}$ insulin alone or
$100 \mathrm{ng} / \mathrm{ml} \mathrm{rh}$ insulin plus ASO-miR-320a. Insulin treatment significantly increased cell viability compared with untreated control cells $(\mathrm{P}<0.05$; Fig. 2A). However, cells in which the expression of miR-320a was inhibited with ASO-miR-320a, cell viability was significantly reduced compared with the insulin group $(\mathrm{P}<0.01$, Fig. $2 \mathrm{~A})$. These results indicated that increased cell viability was induced by insulin treatment, while the administration of ASO-miR-320a inhibited the insulin-induced cell viability.

Colony formation assays were then performed to examine the effect of miR-320a on KGN cell colony formation. Results revealed that insulin treatment significantly increased colony formation compared with untreated control cells $(\mathrm{P}<0.05$; Fig. 2B), while ASO-miR-320a inhibited the effect of insulin on colony formation $(\mathrm{P}<0.05$; Fig. $2 \mathrm{~B})$.

Cell apoptosis was then detected using flow cytometry with FITC-conjugated annexin V/PI staining. The results indicated that insulin administration inhibited cell apoptosis compared with untreated control cells $(\mathrm{P}<0.05$; Fig. 2C). Again, ASO-miR-320a administration counteracted the effect of insulin, resulting in a significantly higher percentage of cell apoptosis in the insulin + ASO-miR-320a group than in the insulin group $(\mathrm{P}<0.05$; Fig. $2 \mathrm{C})$. In view of these results regarding cell apoptosis, mRNA and protein expression levels of Bax and Bcl-2 were detected using RT-qPCR and western blotting assays, respectively (Fig. 2D and E, respectively). The results demonstrated that insulin treatment alone promoted Bcl-2 expression and inhibited Bax expression compared with untreated control cells (Fig. 2D and E). However, administration of ASO-miR-320a reduced the insulin-induced expression of Bcl-2 and insulin-reduced expression of Bax (Fig. 2D and E). This suggested that insulin treatment alone increased the $\mathrm{Bcl}-2 / \mathrm{Bax}$ ratio, and insulin plus ASO-miR-320a treatment reduced the insulin-induced upregulation of the Bcl-2/Bax ratio.

PCGF1 is a target of miR-320a. Targetscan software and microRNA database predicted that PCGF1 was a potential target of miR-320a (Fig. 3A). Followed by verification of this prediction using a Dual-Luciferase reporter assay with vectors containing the WT and mutated PCGF1 3'UTR. Relative luciferase activity was significantly reduced following co-transfection of miR-320a mimics and PCGF1-3'UTR-WT, compared with transfection with PCGF1-3'UTR-WT and control $(\mathrm{P}<0.01$; Fig. 3B); however, there was no significant difference between co-transfection of miR-320a mimics and PCGF1-3'UTR-MUT, compared with transfection with PCGF1-3'UTR-MUT and control P $>0.05$; Fig. 3B). In addition, co-transfection of ASO-miR-320a and PCGF1-3'UTR-WT significantly higher relative luciferase activity than cotransfection ASO-NC and PCGF1-3'UTR-WT $(\mathrm{P}<0.01$; Fig. 3B). However, there was no significant difference between co-transfection of ASO-miR-320a and PCGF1-3'UTR-MUT, compared with transfection with ASO-NC and PCGF1-3'UTR-MUT (P>0.05; Fig. 3B).

PCGF1 mRNA and protein expression levels were then determined in cells with miR-320a expression induced or inhibited, with miR-320a mimics or ASO-miR-320a, respectively. Expression of PCGF1 mRNA and protein reduced following transfection with miR-320a mimics compared 

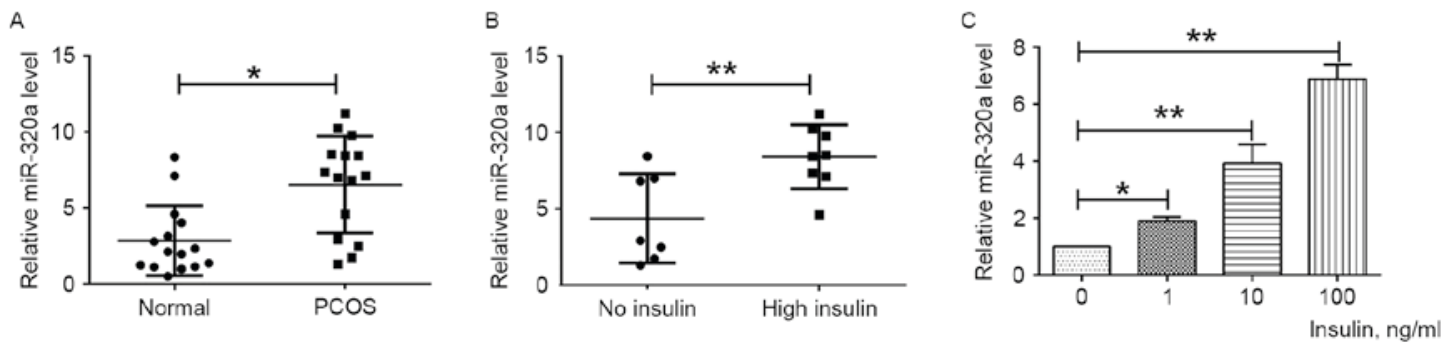

Figure 1. Relative miR-320a expression in PCOS tissues. (A) Relative miR-320a expression in PCOS tissues and corresponding normal tissues. (B) Relative miR-320a expression in patients with different insulin levels. (C) Relative miR-320a expression in KGN cells treated with recombinant human insulin at 0, 1 , $10100 \mathrm{ng} / \mathrm{ml} .{ }^{*} \mathrm{P}<0.05$ and ${ }^{* *} \mathrm{P}<0.01$, as indicated by brackets. miR, microRNA; PCOS, polycystic ovarian syndrome.

A

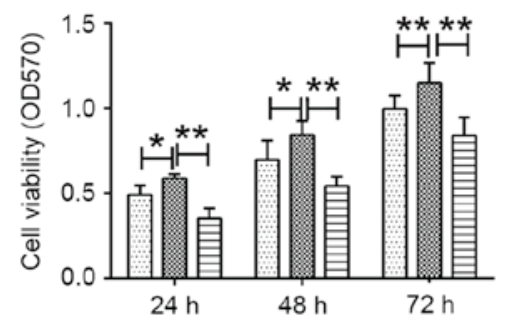

C

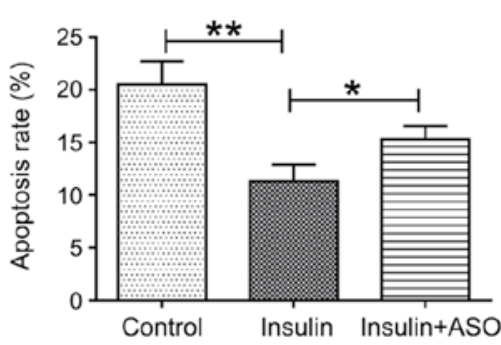

面 Control

Insulin

Insulin+ASO

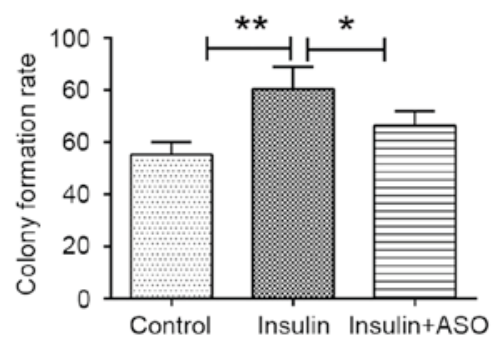

E

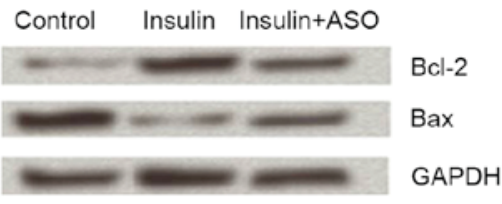

Figure 2. Effects of miR-320a on KGN cell viability, colony formation and apoptosis. Cells were treated with $100 \mathrm{ng} / \mathrm{ml}$ insulin alone or insulin plus ASO-miR-320a. (A) Cell viability was tested by MTS assay. (B) Cell colony formation rate was detected using crystal violet staining. (C) Cell apoptosis was detected by using flow cytometry with fluorescein isothiocyanate-conjugated annexin V/propidium iodide staining. (D) RT-qPCR was used to determine mRNA expression levels of Bcl-2 and Bax. (E) Protein levels of Bcl-2 and Bax were determined by using western blotting. "P<0.05 and ${ }^{* * *} \mathrm{P}<0.01$, as indicated by brackets. miR, microRNA; ASO, antisense oligonucleotide; RT-qPCR, reverse transcription-quantitative polymerase chain reaction; Bcl-2, B-cell lymphoma 2; Bax, Bcl-2 associated protein X.

with control $(\mathrm{P}<0.01$; Fig. $3 \mathrm{C}$ and $\mathrm{D})$, and was upregulated in ASO-miR-320a transfected cells compared with $\mathrm{NC}(\mathrm{P}<0.05$; Fig. 3C and D). A negative relationship between miR-320a and PCGF1 expression was, therefore, confirmed.

PCGF1 inhibits cell viability, colony formation and promotes cell apoptosis. Since PCGF1 was a target of miR-320a and there were a negative relationship between them, it was speculated that PCGF1 expression would modulate KGN cell function as miR-320a inhibition did. Overexpression of PCGF1 was successfully induced via transfection in KGN cells (data not shown), and cell viability was detected in KGN cells were pre-treated with $100 \mathrm{ng} / \mathrm{ml} \mathrm{rh}$ insulin. Results indicated that insulin administration significantly increased cell viability compared with untreated control cells $(\mathrm{P}<0.05$; Fig. 4A), and overexpression of PCGF1 significantly inhibited insulin-induced cell growth ( $\mathrm{P}<0.05$, Fig. 4A). Colony formation assays were then performed to examine the effect of PCGF1 overexpression on KGN cell colony formation: Results revealed that insulin treatment significantly increased colony formation compared with untreated control cells $(\mathrm{P}<0.05$; Fig. 4B), while PCGF1 overexpression inhibited the effect of insulin on colony formation $(\mathrm{P}<0.05$; Fig. 4B). Analysis of cell apoptosis confirmed that cells transfected with PCGF1 expression plasmids plus insulin exhibited a higher percentage of cell apoptosis, than the insulin group ( $\mathrm{P}<0.05$; Fig. $4 \mathrm{C}$ ). Furthermore, RT-qPCR and western blot assays revealed that overexpression of PCGF1 reduced the insulin-induced expression of Bcl-2 and insulin-reduced expression of Bax compared with the insulin group (Fig. 4D and E). This demonstrated that overexpression of PCGF1 contributed to and promoted cell apoptosis, and PCGF1 could partially counteract the action of insulin on cell apoptosis, cell viability and colony formation.

\section{Discussion}

miR-320a has previously been identified as a prognostic factor or potential therapeutic target for tumors $(9,14)$. However, no previous studies have addressed the effect of miR-320a on PCOS. Therefore, to the best of our knowledge, the present 
A

PCGF1 3'UTR WT (64-71)

hsa-miR-320a

PCGF1 3'UTR MUT (64-71)

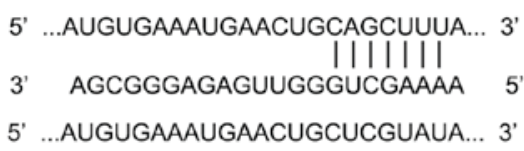

B
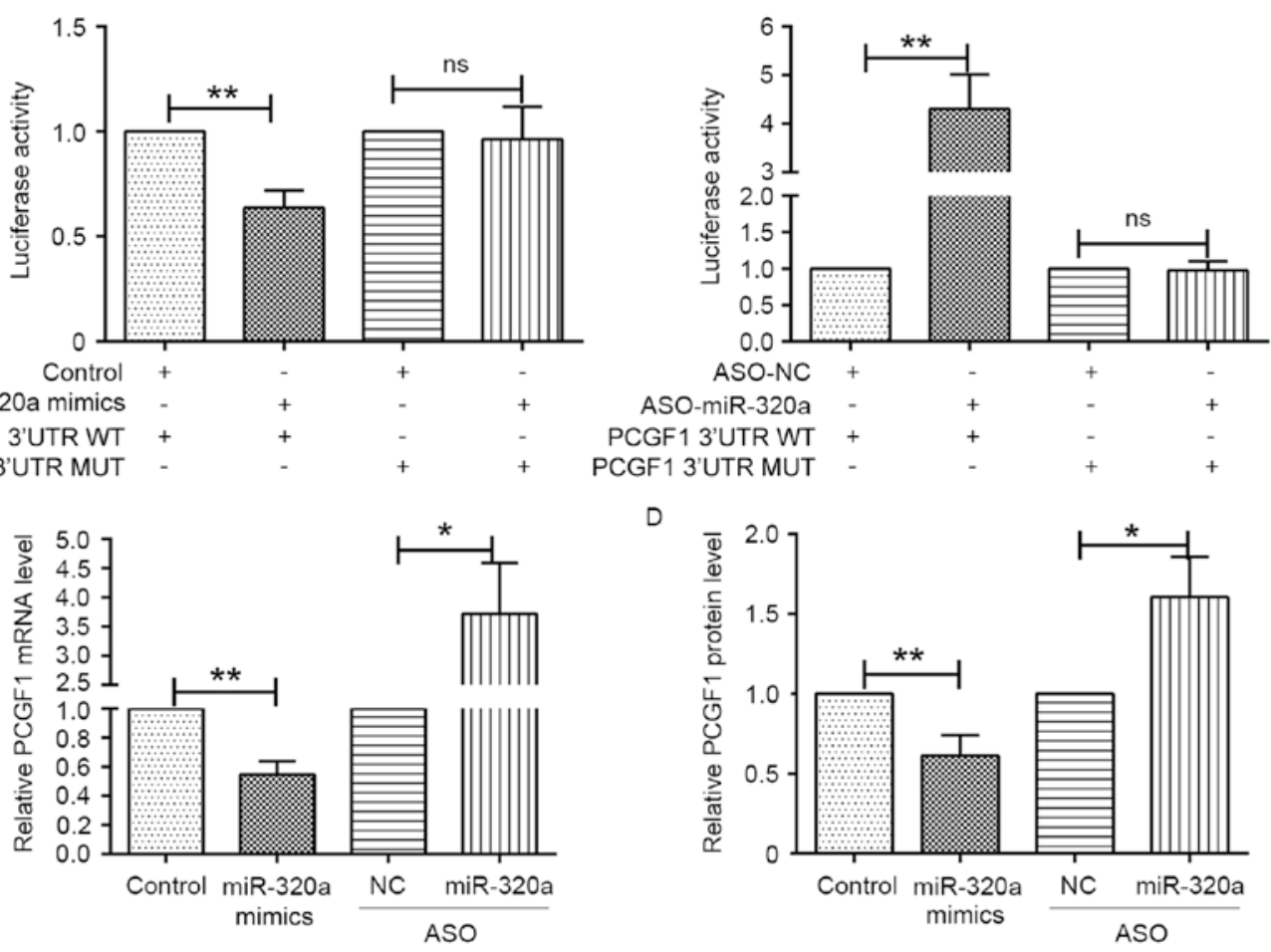

PCGF1

GAPDH

Figure 3. PCGF1 is a target of miR-320a. (A) miR-320a targeting region in PCGF1 was predicted using Targetscan and microRNA database. (B) KGN cells were co-transfected with WT or MUT PCGF1-3'UTR vectors plus miR-320a mimics or control; or ASO-miR-320a or ASO-NC, then luciferase activity was detected by Dual-Luciferase assay. PCGF1 (C) mRNA and (D) protein expression levels were determined by RT-qPCR or western blot, respectively. ${ }^{\text {ns } P>0.05}$, ${ }^{*} \mathrm{P}<0.05$ and ${ }^{* *} \mathrm{P}<0.01$, as indicated by brackets. PCGF1, polycomb group ring finger 1; miR, microRNA; WT, wild type; MUT, mutated; $3^{\prime} \mathrm{UTR}$, $3^{\prime}$ untranslated region; ASO, antisense oligonucleotide; $\mathrm{NC}$, negative control; RT-qPCR, reverse transcription-quantitative polymerase chain reaction.
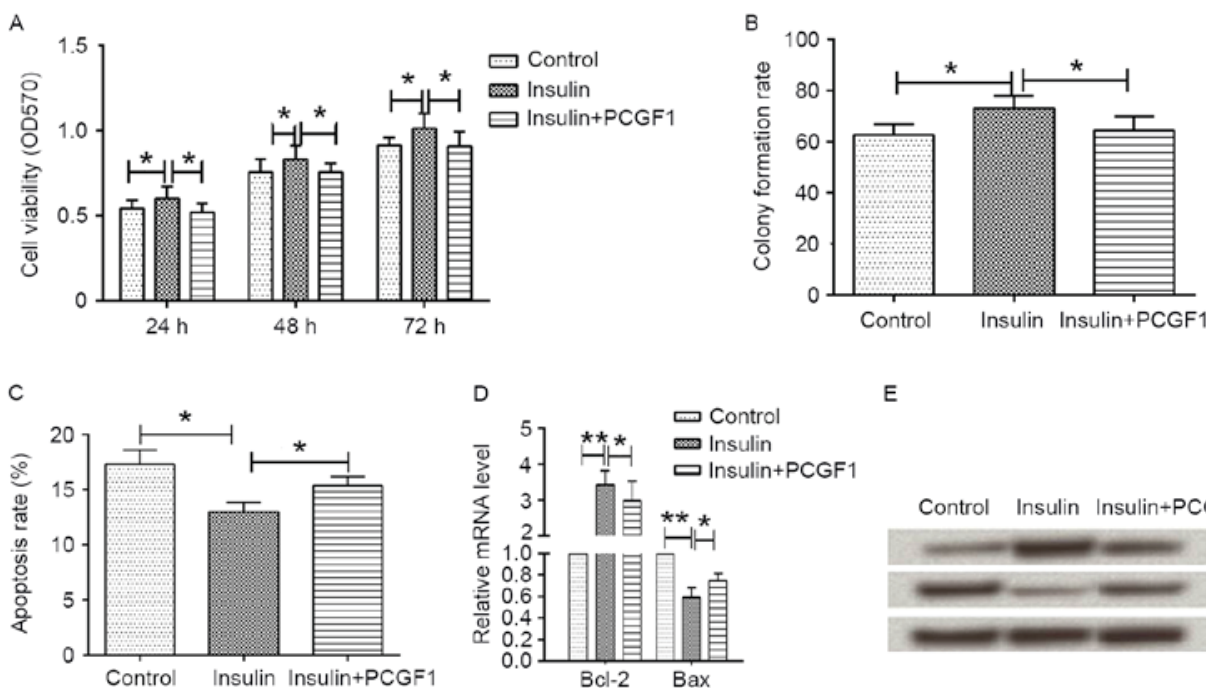

Control Insulin Insulin+PCGF1

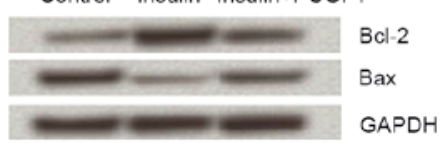

Figure 4. Effects of PCGF1 overexpression on KGN cell viability, colony formation and apoptosis. Cells were treated with $100 \mathrm{ng} / \mathrm{ml} \mathrm{insulin}$ alone or insulin plus PCGF1 expression plasmids. (A) Cell viability was tested by MTS assay. (B) Cell colony formation rate was detected using crystal violet staining. (C) Cell apoptosis was detected by using flow cytometry with fluorescein isothiocyanate-conjugated annexin V/propidium iodide staining. (D) RT-qPCR was used to determine mRNA expression levels of Bcl-2 and Bax. (E) Protein levels of Bcl-2 and Bax were determined by using western blotting. " $\mathrm{P}<0.05$ and ${ }^{* *} \mathrm{P}<0.01$, as indicated by brackets. PCGF1, polycomb group ring finger 1; Bcl-2, B-cell lymphoma 2; Bax, Bcl-2 associated protein X; RT-qPCR, reverse transcription-quantitative polymerase chain reaction. 
study is the first to investigate the role of miR-320a on PCOS by exploring the expression of miR-320a in PCOS tissues as well as the effect on the cell function of KGN granulosa cells. miR-320a was demonstrated to be upregulated in PCOS tissues compared with normal, with expression gradually increasing in an insulin-dependent manner. Furthermore, both inhibition of miR-320a expression via ASO-miR-320a and PCGF1 overexpression reduced cell viability and colony formation and induced cell apoptosis.

miR-320a acts as a prognostic factor of tumors, including colorectal cancer $(10,15)$, hepatocellular carcinoma (16) and gastric cancer (17). Compared with normal controls, expression of miR-320a has been identified to be significantly downregulated in cancers including colon cancer tissues, gastric cancer tissues, salivary adenoid cystic carcinoma and related cancer cell lines $(9,17,18)$; and upregulated in several diseases including breast cancer tissues (19), myelodysplastic syndromes (20), fibromyalgia (21) and laryngeal squamous cell carcinoma (22). These data revealed that miR-320a serves an important role in the progression of at least these cancers or diseases: miR-320a might thus serve as a candidate biomarker of those diseases $(17,23)$. In the present study, significant upregulation of miR-320a in PCOS was identified compared with normal tissues. This may suggest that miR-320a could serve as a biomarker of PCOS.

Insulin resistance is present in $\sim 60-70 \%$ of women with PCOS (4). PCOS with insulin resistance results in differential expression of certain miRNAs, such as miR-93 (3) and miR-223 (24). miR-223 expression in adipose tissues of women with PCOS has also been demonstrated to be positively correlated with insulin resistance, revealing that miR-223 was related to insulin resistance $(24,25)$. In the present study, the expression of miR-320a was confirmed to be insulin-related, with higher miR-320a expression observed in PCOS patients with high insulin levels. This result demonstrated that miR-320a was an insulin resistance related miRNA in PCOS.

miRNAs are regarded as genetic regulators based on their influence and response on gene expression and the associated cell functions (26-28). miRNAs generally exhibit a negative effect on expression of their target genes. For example, upregulation of miR-320a in PCOS tissues is associated with a significant reduction of its verified target, GLUT4 (24). GLUT4 was also a highly predicted target for miR-93, which is also upregulated in PCOS adipose tissues (4). miR-93 and miR-223 have been demonstrated to directly target GLUT4 3'-UTR (4,24). miR-93 has been determined to promote KGN granulosa cell proliferation through targeting of CDKN1A (3). As reported by Jiang et al, expression of miR-93 is upregulated in the PCOS ovarian cortex and, inversely, CDKN1A is downregulated (3). Furthermore, miR-93 overexpression and CDKN1A knockout promote cell proliferation of KGN cells (3). In the present study, the expression of miR-320a was determined to be related to insulin levels. Additionally, inhibition of miR-320a or overexpression of its target PCGF1 reduced insulin-induced cell viability and colony formation. Taken together, the present study demonstrated that cell proliferation promoted by insulin was related to miR-320a and its target PCGF1.

PCGF1 is one of the polycomb-group RING finger homo$\log$ s and is important in the assembly of distinct polycomb repression complex 1 ( $\mathrm{PRC} 1)$ related complexes, which is associated with transcriptional repression and regulation of developmental gene $(29,30)$. PCGF1 has been demonstrated to interact with proteins related to diverse cellular pathways, including regulation of cell cycle and cell proliferation, for example developmental pluripotency associated 4 and PRC1 complexes (30). In the present study, PCGF1 was demonstrated to be a target of miR-320a, and overexpression of PCGF1 and inhibition of miR-320a were demonstrated to inhibit cell proliferation and cell colony formation. In addition, inhibition of miR-320a and overexpression of PCGF1 were demonstrated to reverse the reduction in apoptosis and $\mathrm{Bax} / \mathrm{Bcl}-2$ ratio induced by insulin. Thus, the present study demonstrated that insulin-induced KGN cell proliferation was related to miR-320a through targeting of PCGF1.

In conclusion, expression of miR-320a was upregulated in PCOS tissues compared with normal tissues, and miR-320a expression in PCOS tissues and KGN granulosa cells was demonstrated to be insulin related. Tissues with higher insulin levels, or cells treated with higher insulin, revealed higher miR-320a expression than normal controls. Furthermore, dual-Luciferase reporter assay revealed PCGF1 was a target of miR-320a. Inhibition of miR-320a through ASO-miR-320a inhibited insulin-induced increases in cell viability and cell colony formation, and reversed insulin-induced reductions in cell apoptosis. These data demonstrated that miR-320a may serve as a biomarker of PCOS, and that insulin-induced KGN cell proliferation was related to $\mathrm{miR}-320$ a through targeting PCGF1. The present study may provide a novel insight into the treatment of PCOS. However, the precise functions of miR-320a in PCOS require further investigation.

\section{References}

1. Sørensen AE, Wissing ML, Salö S, Englund AL and Dalgaard LT: MicroRNAs related to polycystic ovary syndrome (PCOS). Genes (Basel) 5: 684-708, 2014.

2. Roth LW, Mccallie B, Alvero R, Schoolcraft WB, Minjarez D and Katz-Jaffe MG: Altered microRNA and gene expression in the follicular fluid of women with polycystic ovary syndrome. J Assist Reprod Genet 31: 355-362, 2014.

3. Jiang L, Huang J, Li L, Chen Y, Chen X, Zhao X and Yang D: MicroRNA-93 promotes ovarian granulosa cells proliferation through targeting CDKN1A in polycystic ovarian syndrome. J Clin Endocrinol Metab 100: E729-E738, 2015.

4. Chen YH, Heneidi S, Lee JM, Layman LC, Stepp DW, Gamboa GM, Chen BS, Chazenbalk G and Azziz R: miRNA-93 inhibits GLUT4 and is overexpressed in adipose tissue of polycystic ovary syndrome patients and women with insulin resistance. Diabetes 62: 2278-2286, 2013.

5. Li Q, Du J, Feng R, Xu Y, Wang H, Sang Q, Xing Q, Zhao X, Jin L, He L and Wang L: A possible new mechanism in the pathophysiology of polycystic ovary syndrome (PCOS): The discovery that leukocyte telomere length is strongly associated with PCOS. J Clin Endocrinol Metab 99: E234-E240, 2014.

6. Murri M, Insenser M, Fernández-Durán E, San-Millán JL and Escobar-Morreale HF: Effects of polycystic ovary syndrome (PCOS), sex hormones, and obesity on circulating miRNA-21, miRNA-27b, miRNA-103, and miRNA-155 expression. J Clin Endocrinol Metab 98: E1835-E1844, 2013.

7. Jiang L, Li W, Wu M and Cao S: Ciculating miRNA-21 as a biomarker predicts polycystic ovary syndrome (PCOS) in patients. Clin Lab 61: 1009-1015, 2015.

8. Yin M, Wang X, Yao G, Lü M, Liang M, Sun Y and Sun F: Transactivation of MicroRNA-320 by MicroRNA-383 regulates granulosa cell functions by targeting E2F1 and SF-1 proteins. J Biol Chem 289: 18239-18257, 2014. 
9. Sun L, Liu B, Lin Z, Yao Y, Chen Y, Li Y, Chen J, Yu D, Tang Z, Wang B, et al: MiR-320a acts as a prognostic factor and inhibits metastasis of salivary adenoid cystic carcinoma by targeting ITGB3. Mol Cancer 14: 96, 2015.

10. Zhao H, Dong T, Zhou H, Wang L, Huang A, Feng B, Quan Y, Jin R, Zhang W, Sun J, et al: miR-320a suppresses colorectal cancer progression by targeting Rac1. Carcinogenesis 35 886-895, 2014.

11. Cheng Z, Qiu S, Jiang L, Zhang A, Bao W, Liu P and Liu J: MiR-320a is downregulated in patients with myasthenia gravis and modulates inflammatory cytokines production by targeting mitogen-activated protein kinase 1. J Clin Immunol 33: 567-576, 2013.

12. Wancket LM, Frazier WJ and Liu Y: Mitogen-activated protein kinase (MKP)-1 in immunology, physiology, and disease. Life Sci 90: 237-248, 2012.

13. Livak KJ and Schmittgen TD: Analysis of relative gene expression data using real-time quantitative PCR and the 2(-Delta Delta C(T)) method. methods 25: 402-408, 2001

14. Sepramaniam S, Armugam A, Lim KY, Karolina DS Swaminathan P, Tan JR and Jeyaseelan K: MicroRNA 320a functions as a novel endogenous modulator of aquaporins 1 and 4 as well as a potential therapeutic target in cerebral ischemia. J Biol Chem 285: 29223-29230, 2010.

15. Zhang Y, He X, Liu Y, Ye Y, Zhang H, He P, Zhang Q, Dong L, Liu Y and Dong J: microRNA-320a inhibits tumor invasion by targeting neuropilin 1 and is associated with liver metastasis in colorectal cancer. Oncol Rep 27: 685-694, 2012.

16. Yao J, Liang LH, Zhang Y, Ding J, Tian Q, Li JJ and He XH: GNAI1 suppresses tumor cell migration and invasion and is post-transcriptionally regulated by $\mathrm{mir}-320 \mathrm{a} / \mathrm{c} / \mathrm{d}$ in hepatocellular carcinoma. Cancer Biol Med 9: 234-241, 2012.

17. Xu Q, Dong QG, Sun LP, He CY and Yuan Y: Expression of serum miR-20a-5p, let-7a, and miR-320a and their correlations with pepsinogen in atrophic gastritis and gastric cancer: A case-control study. BMC Clin Pathol 13: 11, 2013.

18. Sun JY, Huang Y, Li JP, Zhang X, Wang L, Meng YL, Yan B, Bian YQ, Zhao J, Wang WZ, et al: MicroRNA-320a suppresses human colon cancer cell proliferation by directly targeting $\beta$-catenin. Biochem Biophys Res Commun 420: 787-792, 2012

19. Yang H, Yu J, Wang L, Ding D, Zhang L, Chu C, Chen Q, Xu Z, Zou Q and Liu X: miR-320a is an independent prognostic biomarker for invasive breast cancer. Oncol Lett 8: 1043-1050, 2014.
20. Choi JS, Nam MH, Yoon SY and Kang SH: MicroRNA-194-5p could serve as a diagnostic and prognostic biomarker in myelodysplastic syndromes. Leuk Res 39: 763-768, 2015.

21. Bjersing JL, Bokarewa MI and Mannerkorpi K: Profile of circulating microRNAs in fibromyalgia and their relation to symptom severity: An exploratory study. Rheumatol Int 35: 635-642, 2015.

22. Huang J, Lyu H, Wang J and Liu B: MicroRNA regulation and therapeutic targeting of survivin in cancer. Am J Cancer Res 5: 20-31, 2014

23. Hudcova K, Trnkova L, Kejnovska I, Vorlickova M, Gumulec J, Kizek R and Masarik M: Novel biophysical determination of miRNAs related to prostate and head and neck cancers. Eur Biophys J 44: 131-138, 2015.

24. Chuang TY, Wu HL, Chen CC, Gamboa GM, Layman LC, Diamond MP, Azziz R and Chen YH: MicroRNA-223 expression is upregulated in insulin resistant human adipose tissue. J Diabetes Res 2015: 943659, 2015.

25. Zhao C, Liu X, Shi Z, Zhang J, Zhang J, Jia X and Ling X: Role of serum miRNAs in the prediction of ovarian hyperstimulation syndrome in polycystic ovarian syndrome patients. Cell Physiol Biochem 35: 1086-1094, 2015.

26. Nagpal N, Ahmad HM, Molparia B and Kulshreshtha R: MicroRNA-191, an estrogen-responsive microRNA, functions as an oncogenic regulator in human breast cancer. Carcinogenesis 34: 1889-1899, 2013.

27. Fu X, Tian J, Zhang L, Chen Y and Hao Q: Involvement of microRNA-93, a new regulator of PTEN/Akt signaling pathway, in regulation of chemotherapeutic drug cisplatin chemosensitivity in ovarian cancer cells. FEBS Lett 586: 1279-1286, 2012.

28. Iyer A, Zurolo E, Prabowo A, Fluiter K, Spliet WG, van Rijen PC, Gorter JA and Aronica E: MicroRNA-146a: A key regulator of astrocyte-mediated inflammatory response. PLoS One 7: e44789, 2012.

29. Junco SE, Wang R, Gaipa JC, Taylor AB, Schirf V, Gearhart MD, Bardwell VJ, Demeler B, Hart PJ and Kim CA: Structure of the polycomb group protein PCGF1 in complex with BCOR reveals basis for binding selectivity of PCGF homologs. Structure 21: 665-671, 2013.

30. Oliviero G, Munawar N, Watson A, Streubel G, Manning G, Bardwell V, Bracken AP and Cagney G: The variant polycomb repressor complex 1 component PCGF1 interacts with a pluripotency sub-network that includes DPPA4, a regulator of embryogenesis. Sci Rep 5: 18388, 2015. 